\title{
COVID-19 Guidelines Changing Faster than the Virus: Implications of a Clinical Decision Support App
}

Yair Saperstein ${ }^{1}$, Shawn Y. Ong ${ }^{2}$, Tarik Al-Bermani' ${ }^{1}$, Joongheum Park ${ }^{3}$, Yiela Saperstein ${ }^{4}$, Jadesola Olayinka ${ }^{1}$, Anjali Jaiman ${ }^{1}$, Andrew Winer ${ }^{5}$, Moro O. Salifu ${ }^{1}$ and Samy I. McFarlane ${ }^{1{ }^{*}}$

${ }^{1}$ Department of Medicine SUNY-Downstate-Health Science University, Brooklyn, New York, United States

${ }^{2}$ Emergency Medicine, Yale University, New Haven, United States

${ }^{3}$ Beth Israel Deaconess Medical Center, Harvard University, Cambridge, United States

${ }^{4}$ Department of Biomedical Engineering, Columbia University, New York, United States

${ }^{5}$ Kings County Hospital Center, Brooklyn, New York, United States

\section{Abstract}

Publication History:

Novel coronavirus disease (COVID-19) is a pandemic affecting over 10 million people in 160 countries. Received: June 30, 2020 Its spread, and the medical communities' response, cast light on an important deficiency in the speed and Accepted: July 13, 2020 effectiveness for evidence-based recommendations to reach the bedside in academic medical practice. Published: July 15, 2020 We built a clinical decision support tool on the avoMD platform that systematizes and personalizes the treatment of COVID-19 by bringing point-of-care access to the guidelines specific to individual cases to the clinician's hands. This app has the potential to improve the mortality for COVID-19.

\section{Keywords:}

COVID-19, Decision support app, Participatory design

\section{Introduction}

Novel coronavirus disease (COVID-19) is a pandemic that since its discovery in Wuhan, China in December 2019 has reached over 10 million people [1], in over 160 countries, with a case-fatality rate of $4.9 \%$ in the United States and a much higher $11-16 \%$ in countries including Italy (14.5\%), Spain (11.4\%), France (14.8\%), United Kingdom (13.9\%), and Belgium (15.9\%) [2].

As the number of patients in each locale exponentially increases, strained medical systems struggle. One manifestation of the struggle is designing, using and applying clinical decision treatment algorithms using the evidence-based medicine paradigm [3]. Evidence-based medicine relies on well structured, well researched rationales to guide clinical decision making. In the case of COVID-19, as a novel disease, there was limited clinical knowledge when it hit the United States, and the classic evidence-based medicine could not keep pace with the rapid on-the-ground evolution of knowledge about the disease and its management.

Thus, many clinicians and physician groups relied on a socialmedia based crowdsourced sharing based on the experiences of fellow clinicians in other countries [4]. These large Facebook groups allowed clinicians to share their experiences and answer each other's' questions [5]. Even so, effectively synthesizing the information requires the development and dissemination of practice guidelines [6]. The CDC produced interim guidance for the treatment of COVID-19 [7] as did the WHO [8] and IDSA [9], however with the rapidly changing guidelines, it has been a struggle to disseminate to clinicians the changing information in the form of a treatment guideline, leading to the sharing of protocols through collaborative methods including social media and a shared Google doc [10]. This is as medical systems struggled with and continue to struggle with the production of guidelines for clinicians in a manner that is reliable, current, and ensures that clinicians have access to them at the point of care. Indeed, the utility of clinical guidelines is only in their practical implementation [11-13]. Even with clinical practice guidelines available, the time, effort and skills needed to access these guidelines are not available to everyone $[14,15]$.
In this editorial, we introduce a new type of clinical decision support (CDS) tool for COVID-19, which provides clinicians a subset of the COVID-19 guidelines personalized to the clinical features at the point-of-care. In contrast to the resources on social media, on the shared Google doc, and on the individual websites of the CDC, WHO, and IDSA, our tool aims to be accessible at the point of care. On the other hand, in contrast to existing CDS tools that automate decisions or order sets $[16,17]$, we intended that the tool helps clinicians make informed decisions rather than make passive decisions. This allows the clinicians to benefit from individualized education. Finally, we describe how our app (built on the avoMD platform) can be updated and reviewed in real-time to allow for new evidence and guidelines to be integrated. Our app has the potential of improving the mortality of COVID-19.

\section{Framework Design: Interactive Guideline with Participatory Design}

Our team built an interactive text-based platform in which each paragraph; sentence or summary item is activated or deactivated by triggers based on user inputs. The user chooses among button choices and inputs numeric values on the first screen, and the inputs determine the activation of the triggers.

We used Google App Script to build a spreadsheet-based tool ('editing app') where the editing experts ('authors') can define data entry elements (e.g., segmented buttons, pickers, numeric or text

*Corresponding Author: Prof. Samy I. McFarlane, College of Medicine, Department of Medicine, Division of Endocrinology, Internal Medicine Residency Program Director, State University of New York, Downstate Medical Center, 450 Clarkson Ave, Box 50, Brooklyn, New York, USA, Tel 718-270-6707, Fax 718-2704488; E-mail: smcfarlane@downstate.edu

Citation: Saperstein Y, Ong SY, Al-Bermani T, Park J, Saperstein Y, et al. (2020) COVID-19 Guidelines Changing Faster than the Virus: Implications of a Clinical Decision Support App. Int J Clin Res Trials 5: 148. doi: https://doi. org/10.15344/2456-8007/2020/148

Copyright: (C) 2020 Saperstein et al. This is an open-access article distributed under the terms of the Creative Commons Attribution License, which permits unrestricted use, distribution, and reproduction in any medium, provided the original author and source are credited. 
data field, multiple-item selectors, or multimedia inputs) for the data inputs. The editing authors are allowed to set triggers to each data entry item, allowing branching of questions and the end-users being exposed only to a subset of questions at each usage. The expert authors can name and assign variables to specific questions (e.g., the answer to the question of the presence of symptoms can be saved to a variable named 'symptoms'). The authors can also assign boolean triggers to the text contents as we discussed above.

When the authors finish editing the contents and decide to update a clinical module, the app script packages the contents into multiple CSV files (dashboard, pages, contents of each page, and references) and sends the files to Google Firebase, which is Google's real-time database and backend for mobile apps. We developed the front-end side of the app service in React Native.

Twenty clinicians without programming skills tested the editing app to build drafts of test apps for several clinical problems and to improve participatory aspects of the framework. A senior public health researcher and infectious disease expert reviewed and edited all of the contents of the app at its first publication, and the app is continuously edited on a weekly basis for both accuracy and ease of understanding.

\section{Development of the COVID-19 Protocol}

Guidelines developed for COVID-19 by the Centers for Disease Control and Prevention (CDC) [18], World Health Organization (WHO) [19], Society of Critical Care Medicine (SCCM) [20], and the Infectious Disease Society of America (IDSA) [21] were used as the basis of our decision framework. The overall structure of the decision framework was guided by clinical workflow for both inpatient and outpatient settings and patient conditions. Informational buttons are provided that guide clinicians on presenting symptoms and considerations for suspicion of SARS CoV-2 infection. The corresponding medical society is noted for each guideline so users can easily determine the origin along with links to specific online references.

\section{First iteration of the guidelines}

As a novel virus, COVID-19 presented unique challenges, including a shifting focus of guidelines. The initial goal of these guidelines was the early identification and isolation of suspected cases to prevent further spread. Thus, at that time, our tool utilized the WHO and CDC guidelines to determine which symptomatic patients met criteria for being a Persons Under Investigation requiring testing and isolation, and offered an algorithmic tool that allowed the clinician to perform quick risk stratification so that the most up to date recommendations could be provided. At that time, the algorithm determined whether or not patients had symptoms that were consistent with COVID-19 (fever, cough, shortness of breath, at that time) plus epidemiological risk factors (exposure to COVID-19 positive persons or suspicious travel history, at that time). This was created as a calculator, where, if positive, the patient was deemed to be a Persons Under Investigation (PUI) which required immediate isolation and recommended testing.

The algorithm regarding patients who were asymptomatic involved more history taking. The asymptomatic individuals were split up into health care personnel and general population. Given the nature of the work, health care personnel were at higher risk at baseline. The factors that affected risks status were mainly Personal Protective Equipment being worn properly and involvement in a high risk procedure (defined as intubation, CPR, bronchoscopy). The general population risk factors were mainly travel history or exposure to known symptomatic COVID-19 positive patients. Using the CDC guidelines, our tool was able to create a stepwise fashion in which providers could screen these asymptomatic general population with exposures to identify their risk factors. At the end of each algorithm, the CDS tool appropriately placed a risk level defined as "No Identifiable Risk", "Low Risk", "Medium Risk", or "High Risk". Within these final pages, recommendations based on risk level were made regarding testing, quarantine, monitoring for symptoms with or without health department supervision, recommendations for travel and work. The final pages also provided recommendations on how specimens should be obtained and delivered for testing.

Testing was initially sparse meaning that resources were to be allocated to those who were at a certain risk level of testing positive for COVID-19. As prevalence increased, the recommendations published by the CDC, WHO, IDSA and SCCM evolved to meet these demands. Testing capability increased and requirements for screening became less stringent. We adapted our tool on a daily basis to match these shifting guidelines.

\section{Latest iteration of the guidelines}

As the guidelines expanded to include treatment modalities, we modified our app to match this expansion. In the latest version, the clinician is guided through high suspicion, low suspicion, and consideration of a false negative test result (Figure 1). For the hospital setting, the user is guided through recommendations for patient management and infection control from the previously mentioned medical societies. The guidance covers topics of patient placement and transportation, transmission-based precautions, oxygen therapy, use of corticosteroids, and experimental treatment options such as remdesivir, hydroxychloroquine, and lopinavir/ritonavir.

For patients who are in the intensive care unit (ICU), users are presented with a link to calculate the Sequential Organ Failure Assessment (SOFA) score, a mortality prediction score that is based on the degree of dysfunction of six organ systems [21]. ICU care guidance is provided to the clinician based on SCCM guidelines covering topics such as intubation recommendations, ventilator settings, and acute respiratory distress syndrome (ARDS) management (Figure 2).

Finally, for the patients who are being discharged to home or are determined capable of managing their illness at home, guidance is provided for both test based and non-test based strategies from the CDC.

\section{Discussion and Future Directions}

Guidelines have been changing rapidly throughout the course of the COVID-19 pandemic, and the classical methods of scholarly publication were not sufficient for guiding point-of-care decisions. To our knowledge, there are no currently existing apps that guide clinicians through the process of COVID-19 management while providing education. Existing systems attempt to use either static informational pages (e.g., guidelines or UpToDate), calculators (e.g., MDCalc), or automatic order sets or decisions to help improve access to and implementation of guidelines. Our tool is unique in that it delivers tailored aspects of the management with additional information specific to individual clinical scenarios at the point of care. In doing so, it not only provides clinical decision support, 


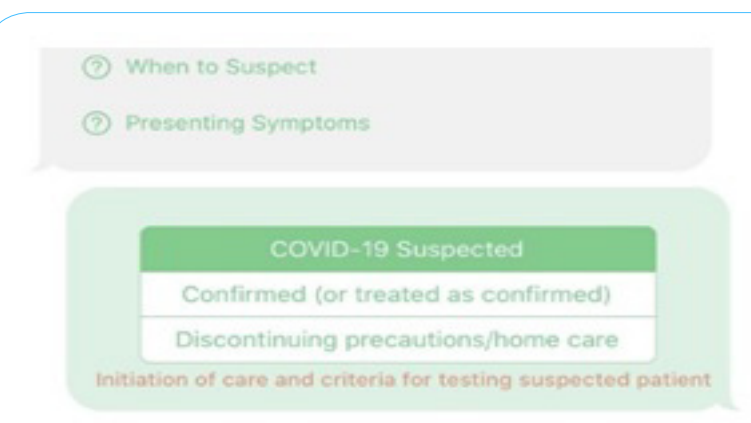

\section{Suspected for COVID-19}

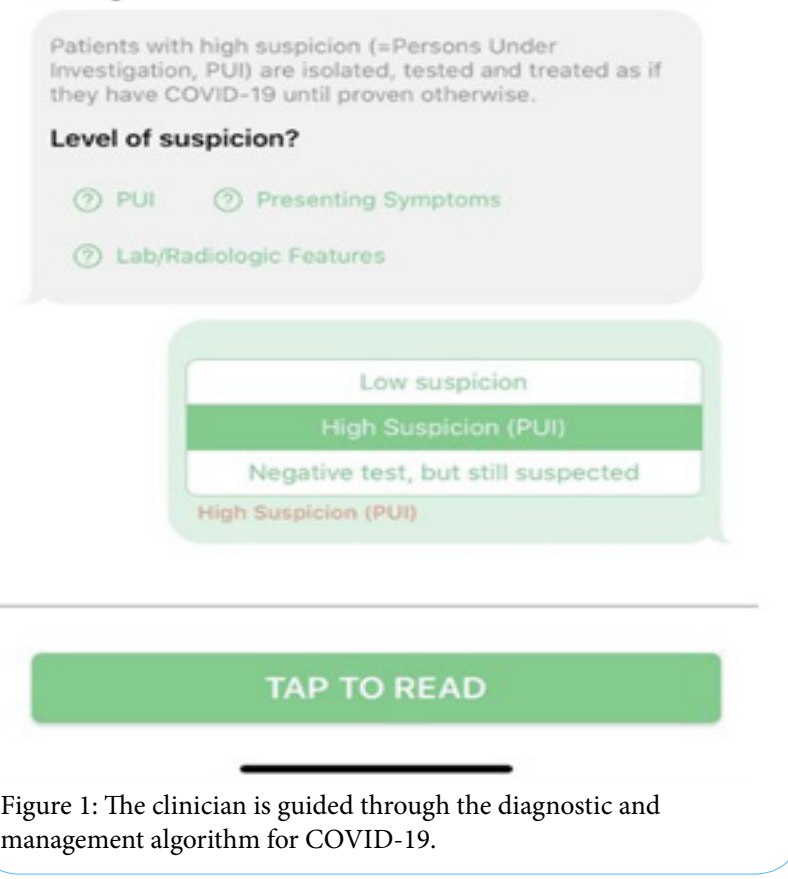

but is a resource for continual education for the clinician. Our app is free to download on both the Android and the Apple App Store ("avoMD"). Since it currently does not link or store any personal patient data, there are no HIPPA concerns. While this our app can potentially improve care and decrease mortality, further testing is required for confirmation.

We have conducted formal real-world validation by means of measuring the systemic usability scale (SUS), physician satisfaction and decision understanding at the beginning stage of our implementation and used participatory design to improve the interface of our app based on input (paper submitted for publication). We will trial the implementation of our app in a hospital setting and use an interrupted time-series design to assess its effects with each clinician as a target of randomization. Since our app provides easier access to guidelines, rather than a promotion of unique guidelines, the ethical concerns are minimal, similar to existing guideline repositories. If successful, we will spread awareness through physician communities by publishing our evaluation results. We will also ask users to rate our app based on ease of use, usefulness of the tool, usefulness of the content, and overall satisfaction. This optional survey will ask for comments, as well, helping us to improve the tool.

Finally, our tool may be applied to a vast array of diseases and may change the paradigm for consumption and use of clinical guidelines.

\section{Contents}

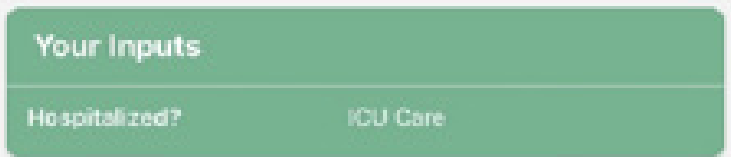

\section{Summary}

ICU Care

- Negative pressure room/respirator masks are recommended for aerosol-generating procedures.

- Using video-guided laryngoscopy is recommended for endotracheal intubation to minimize the risk of transmission.

= Vt 4-8 mllkg and Pplat $<30 \mathrm{~cm} \mathrm{H} 2 O$ for ARDS. Consider higher PEEP, NMBA boluses, Prone ventilation $12-16 \mathrm{~h}$ and NMBA infusion for $24 \mathrm{~h}$ for severe ARDS.

= Use of corticostercid is discouraged for ventilated adults (without ARDS)

- Consider corticosteroid for ARDS/COVID-19

= SCCM suggests AGAINST the routine use of IVIG and convalescent plasma.

= SCCM suggests AGAINST the routine use of Iopinavirfritonavir. Limited evidence for issuing recommendations for chloroquir hydroxychloroquine and remdesivir.

Figure 2: Guidance is provided to the clinician based on society guidelines covering topics including intubation recommendations, ventilator settings, and acute respiratory distress syndrome (ARDS) management.

\section{Conclusion}

COVID-19 highlights an issue with our current process of sharing protocols and research: it is not practically accessible at the pointof-care in a way that is efficient enough to keep up with the virus. We built a clinical decision support tool that systematizes and personalizes the treatment of COVID-19 by bringing point-of-care access to the guidelines specific to individual cases to the clinician's hands. By bringing standard of care guidelines to every clinician, this app has the potential to improve the mortality for COVID- 19 .

\section{Acknowledgements}

This work is supported, in part, by the efforts of Dr. Moro O. Salifu M.D., M.P.H., M.B.A., M.A.C.P., Professor and Chairman. 


\section{Competing Interests}

The authors declare that they have no competing interests.

\section{References}

1. https://www.worldometers.info/coronavirus/

2. https://coronavirus.jhu.edu/data/mortality

3. Guyatt G, Cairns J, Churchill D, Cook D, Haynes B, et al (1992) EvidenceBased Medicine: A New Approach to Teaching the Practice of Medicine. JAMA 268: 2420-2425.

4. https://blogs.scientificamerican.com/observations/why-social-media-iscrucial-for-frontline-physicians-in-the-fight-against-covid-19/

5. https://www.bloomberg.com/news/articles/2020-03-24/covid-19-mysteriesyield-to-doctors-new-weapon-crowd-sourcing

6. Edwards DJ (2015) Dissemination of Research Results: On the Path to Practice Change. Can J Hosp Pharm 68: 465-469.

7. https://www.cdc.gov/coronavirus/2019-ncov/hcp/clinical-guidancemanagement-patients.html

8. https://www.who.int/emergencies/diseases/novel-coronavirus-2019/ technical-guidance

9. https://www.idsociety.org/practice-guideline/covid-19-guideline-treatmentand-management/

10. https://www.newyorker.com/news/q-and-a/inside-a-new-effort-to-createguidelines-for-treating-covid-19-patients

11. Aujesky D, Fine MJ (2005) Does guideline adherence for empiric antibiotic therapy reduce mortality in community-acquired pneumonia? Am J Respir Crit Care Med 172: 655-656.

12. Bero LA, Grilli R, Grimshaw JM, Harvey E, Oxman AD, et al. (1998) Closing the gap between research and practice: an overview of systematic reviews of interventions to promote the implementation of research findings. The Cochrane Effective Practice and Organization of Care Review Group. BMJ 317: 465-468.

13. Biesbroek S, Verschuren WMM, Boer JMA, E van de Kamp M, T van der Schouw Y, et al. (2017) Does a better adherence to dietary guidelines reduce mortality risk and environmental impact in the Dutch sub-cohort of the European Prospective Investigation into Cancer and Nutrition? $\mathrm{Br}$ J Nutr 118: 69-80.

14. Cabana MD, Rand CS, Powe NR, Wu AW, Wilson MH, et al. (1999) Why don't physicians follow clinical practice guidelines? A framework for improvement. Jama 282: 1458-1465.

15. Saperstein Y, Park J, McFarlane SI (2018) Closing the Mortality Gap in Diabetic Ketoacidosis and Hyperosmolar Hyperglycemic State: Implications of a Clinical Decision Support App. Int J Clin Endocrinol Metab 4: 8-11.

16. Bonis PA, Pickens GT, Rind DM, Foster DA (2008) Association of a clinical knowledge support system with improved patient safety, reduced complications and shorter length of stay among Medicare beneficiaries in acute care hospitals in the United States. Int J Med Inform 77: 745-753.

17. Joyner Blair AM, Hamilton BK, Spurlock A (2018) Evaluating an Order Set for Improvement of Quality Outcomes in Diabetic Ketoacidosis. Adv Emerg Nurs J 40: 59-72.

18. https://www.cdc.gov/coronavirus/2019-nCoV/hcp/index.html

19. https://www.who.int/emergencies/diseases/novel-coronavirus-2019/ technical-guidance

20. https://www.sccm.org/getattachment/Disaster/SSC-COVID19-CriticalCare-Guidelines.pdf

21. https://www.idsociety.org/practice-guideline/covid-19-guideline-treatmentand-management/

22. Vincent JL, Moreno R, Takala J, Willatts S, De Mendonça A, et al. (1996) The SOFA (Sepsis-related Organ Failure Assessment) score to describe organ dysfunction/failure. On behalf of the Working Group on SepsisRelated Problems of the European Society of Intensive Care Medicine. Intensive Care Med 22: 707-710. 